\title{
Integration of thermophysiological body model in CFD
}

Research Article

\author{
George Pichurov ${ }^{1 *}$, Peter Stankov ${ }^{1}$ \\ 1 Deptartment of Hydroaerodynamics and Hydraulic Machines, Technical University of Sofia, Bulgaria
}

Received 11 December 2012; accepted 13 April 2013

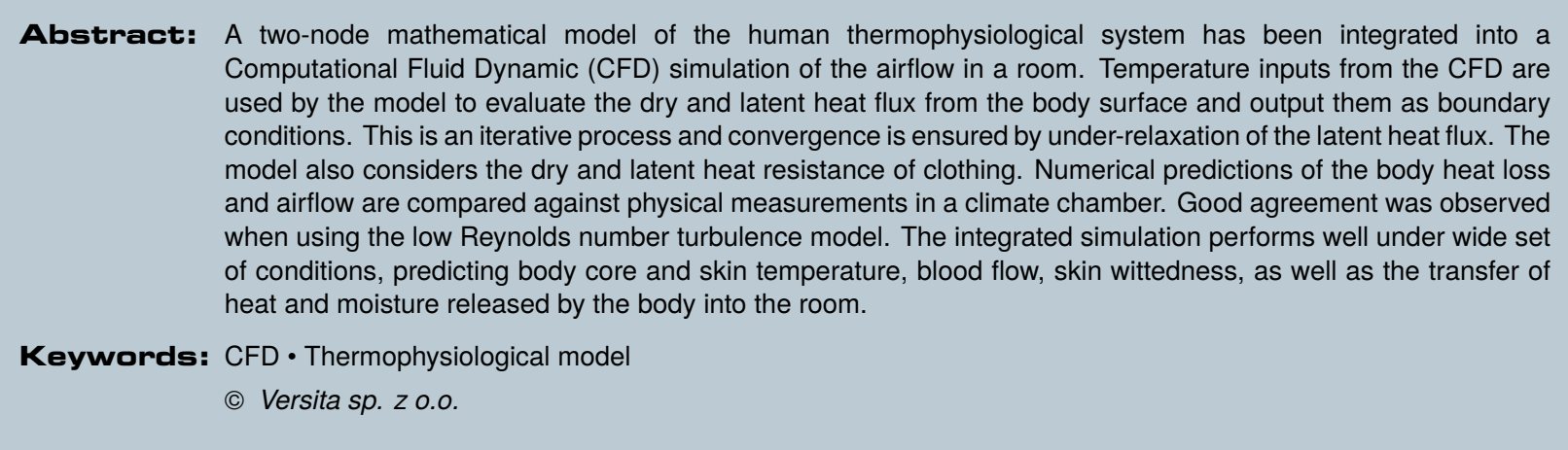

\section{Introduction}

The thermal sensation of occupants in enclosures has been a subject of study for many years. The numerical approach to the problem involves calculation of the surrounding airflow and temperature distribution and then prediction of the likelihood for thermal comfort at that particular location. Researchers have recently noted that the human body is not a passive source of heat, but that it generates different amounts of heat dependent on both the metabolic activity and the thermal conditions of the room. Since room thermal conditions depend on the heat sources from humans, this becomes an interrelated problem requiring integrated approach.

The thermal behavior of a human body in a given

*E-mail: jorence@abv.bg environment is predicted by a thermophysiological model. A thermophysiological model (TPM) has the purpose of predicting the distributed thermal parameters in the human body (core and skin temperature, dry and latent heat loss from body surface, blood flow, etc.) based on the external environmental parameters (temperature, air velocity, radiant temperature, air humidity) and personal factors affecting heat generation (level of activity) and heat loss (clothing insulation). The evolution of such models began around the 1960s with the work of [1]. Later, [2] developed a robust and computationally efficient model that reasonably simplifies the human thermophysiological system. This model found place in [3] and was also selected for use in the present numerical study.

Such models can be used either independently to predict thermal sensation in fixed preselected environments, or integrated into numerical simulation of the airflow. [4] successfully integrated the original model [2] into a numerical simulation of the airflow for a naked human 
body. In the present study the model has been adapted to accommodate a clothed human body. Integration of a clothed human body model in a CFD simulation has also been reported by [5]. More advanced multi-segmented models, based on the work [6], have been reported by [7]. The main advantage of the model employed here is its simplicity and the ability to integrate it into a commercial CFD package with minimum effort and without a thirdparty software. This study also presents the conditions necessary for convergence of the integrated simulation, so that the algorithm can be successfully used by others.

\section{Implementation of the thermo- physiological model in CFD}

The thermo-physiological model of [2], also adopted and described in [3], approximates the human body by two concentric layers - core and skin. Heat is generated in the core through metabolism and is transferred to the environment through pulmonary exhalation and to the skin layer through thermal conduction and blood circulation. The heat gained by the skin layer is transferred from the body surface to the environment as dry heat loss due to convection and radiation and latent heat loss due to moisture evaporation. The energy conservation equations for the two layers under steady state conditions can be written as:

$$
\begin{gathered}
M-W-\left(C_{\mathrm{res}}+E_{\mathrm{res}}\right)-Q_{\mathrm{cr}, \mathrm{sk}}=0 \text { core } \\
Q_{\mathrm{cr}, \mathrm{sk}}-\left(C+R+E_{\mathrm{sk}}\right)=0 \text { skin }
\end{gathered}
$$

$M$ is rate of metabolism, $\left[\mathrm{W} / \mathrm{m}^{2}\right], W$ is mechanical work, $\left[\mathrm{W} / \mathrm{m}^{2}\right], C_{\text {res }}$ is dry heat loss of exhalation, $\left[\mathrm{W} / \mathrm{m}^{2}\right], E_{\text {res }}$ latent heat loss from exhalation, $\left[\mathrm{W} / \mathrm{m}^{2}\right], Q_{\mathrm{cr}, \mathrm{sk}}$ is heat transferred between core and skin layer, $\left[\mathrm{W} / \mathrm{m}^{2}\right], C+R$ is the dry heat flux from body surface due to convection and radiation, $\left[\mathrm{W} / \mathrm{m}^{2}\right], E_{\mathrm{sk}}$ is latent heat loss from body surface, $\left[\mathrm{W} / \mathrm{m}^{2}\right]$. The units of the parameters involved are heat flux per unit of body surface area.

The rate of metabolism, $M$, is a tabulated quantity available for different physical activities. The mechanical work, $W$, is usually assumed to be zero, recognizing that almost all the human physical effort is converted into heat. Relationships for calculating the rest of the quantities in eq. (1) and (2) are described in [4] and [8]. The implementation of the model into a numerical simulation is described below with the most important steps outlined. After the exhalation heat losses have been calculated, the dry heat loss from the body surface and the surface temperature are determined from the CFD package and

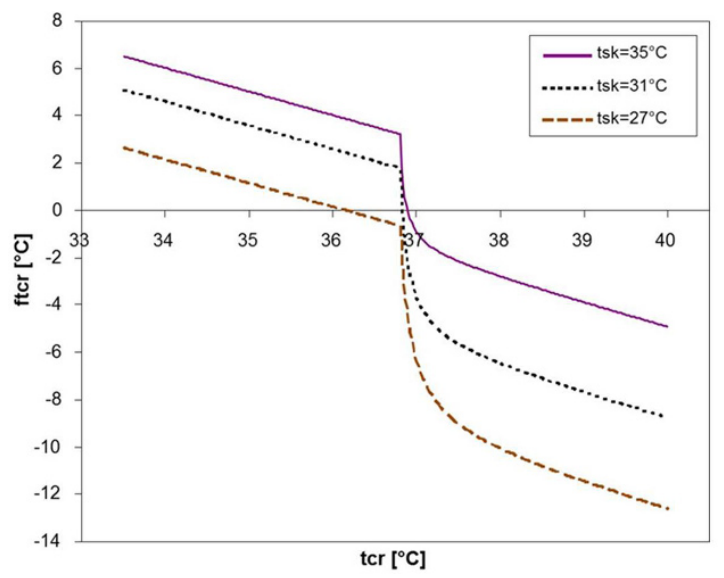

Figure 1. Roots of the body core temperature for different skin temperatures.

imported as data into the model. The skin temperature is then calculated by the Fourier law of thermal conductivity:

$$
t_{\mathrm{sk}}=t_{\mathrm{cl}}+(C+R) R_{\mathrm{cl}}
$$

$t_{\mathrm{sk}}$ is skin temperature $\left[{ }^{\circ} \mathrm{C}\right], t_{\mathrm{cl}}$ is external clothing temperature $\left[{ }^{\circ} \mathrm{C}\right]$, imported from CFD, C $+\mathrm{R}\left[\mathrm{W} / \mathrm{m}^{2}\right]$ is dry heat flux, also imported from CFD, $R_{\mathrm{cl}}$ is dry heat resistance of clothing $\left[\mathrm{m}^{2} \mathrm{~K} / \mathrm{W}\right]$. Calculating the skin temperature enables an implicit equation for the body core temperature (see $[4,8]$ ) to be solved by interval halving [8]. The implicit functional relationship for body core temperature depends on skin temperature, as shown in Figure 1. The body core temperature for a particular skin temperature lies on the crossing point of the curve with the $x$-axis. To ensure convergence, the initially selected interval of body core temperatures must be wide enough to enclose the root of the equation for a wide range of skin temperatures. Since the surface temperature (and thus the skin temperature according to eq. (3)) will exhibit large and unrealistic variations until convergence of the CFD iterations, a wider initial range of body core temperatures is recommended; a minimum lower value of $20^{\circ} \mathrm{C}$ and a maximum upper value of $45^{\circ} \mathrm{C}$.

After calculating the body core temperature using the algorithm above, it is necessary to determine the latent heat flux $E_{\text {sk }}$ from the body surface. This work proposes a novel solution by applying underrelaxation of the latent heat flux at each iteration of the thermophysiological model:

$$
E_{\mathrm{sk}}^{i+1}=E_{\mathrm{sk}}^{i}+\left(E_{\mathrm{sk}}^{i+1}-E_{\mathrm{sk}}^{i}\right) \alpha
$$


where $\alpha$ is the relaxation coefficient and upper indices indicate the iteration number. This important modification was necessary to achieve stability of the integrated simulation. A value of the relaxation coefficient of 0.1 will suffice for most combinations of metabolic rate, clothing insulation and room conditions. However, for extreme values of these quantities it is necessary to use a lower value for $\alpha$.

After the latent heat loss has been assigned in the manner detailed above, the boundary conditions for the successive CFD iteration are calculated. The boundary conditions are dry heat flux and moisture flux. The dry heat flux for each cell of the body surface is calculated by combining equation (1) and equation (2):

$$
C+R=M-E_{\mathrm{res}}-C_{\mathrm{res}}-E_{\mathrm{sk}}
$$

and the moisture flux is calculated by:

$$
m_{\mathrm{sk}}=E_{\mathrm{sk}} / h_{\mathrm{fg}}
$$

where $h_{\mathrm{fg}}$ is the latent specific heat of water at $30^{\circ} \mathrm{C}$ and equal to $2430 \mathrm{~kJ} / \mathrm{kg}$.

\section{Geometry and boundary conditions}

The geometry and boundary conditions simulated in this study correspond to a climate chamber used in a validation experiment described later. A seated human body mannequin is located in the middle of a $2.44 \times 1.2 \times 2.46 \mathrm{~m}$ chamber, facing a uniform airflow with an average velocity of $0.27 \mathrm{~m} / \mathrm{s}$ and temperature range $19.6^{\circ} \mathrm{C}-20.8^{\circ} \mathrm{C}$ (Figure 2). There are two circular $0.25 \mathrm{~m}$ exhausts on the wall behind the mannequin, located $0.6 \mathrm{~m}$ from the floor and ceiling, respectively.

To model the human body, the geometry of a thermal mannequin was scanned topographically and discretized into STL file format as 125000 triangular facets. The vast majority of these pertain to the knees, which were wrapped in aluminium foil and produce numerous wrinkles and unnecessary detail (Figure 3a). To overcome this issue, the surface was simplified with a software tool named VRMesh and the surface representation was reduced almost tenfold to 13000 facets without losing detail (Figure 3b).

The mannequin body model was split into 16 parts at pre-processing level in accordance with the body parts of the original design. A triangular computational mesh was generated with the cell size dynamically adapted so

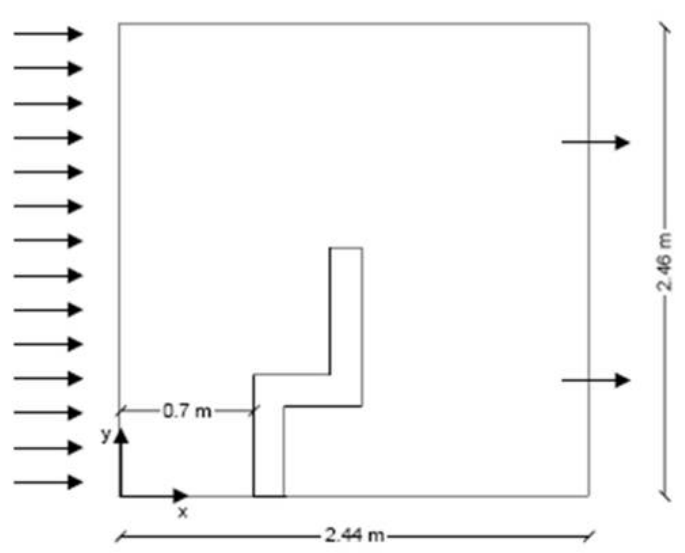

Figure 2. Roots of the body core temperature for different skin temperatures. (a)

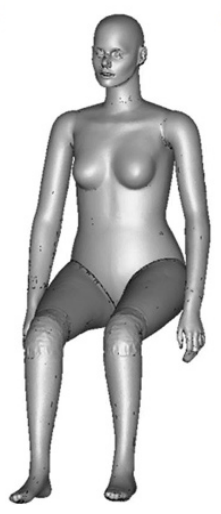

(b)

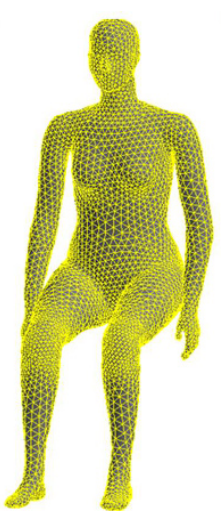

(c)

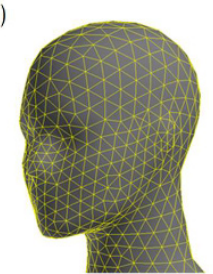

Figure 3. Mannequin geometry and computational mesh. (a) Original geometry; (b) Simplified geometry and body mesh; (c) Face mesh.

that the angle between normals of two neighboring cell faces did not exceed $15^{\circ}$. This generates denser mesh when the curvature of the surface changes rapidly, as seen by the facial mesh on Figure 3c. A pilot simulation was made to determine the dimensionless wall distance, $y^{+}$, around the mannequin surface, and the volumetric mesh was refined where necessary to limit this distance to 5 in accordance with the requirements of the selected LRN turbulence model. This was achieved for all body parts except the back and pelvis, for which $y^{+}$was on the order of 10-15. The computational mesh of the room consists of nearly 670000 tetrahedral elements refined near the 


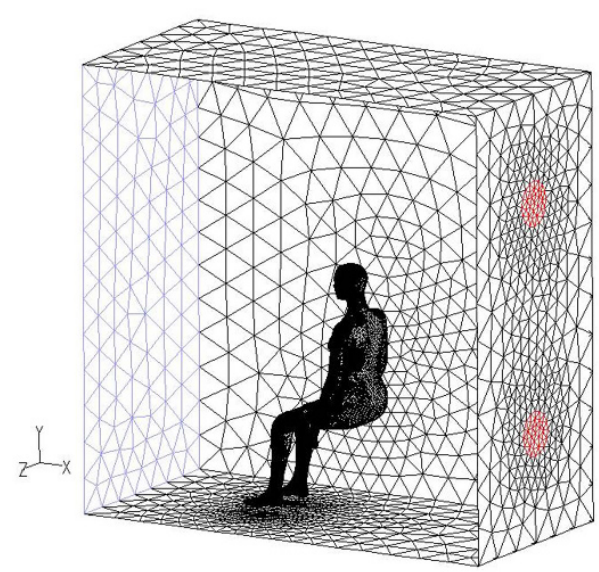

Figure 4. Computational mesh composed of tetrahedral elements.

mannequin surface and is shown on Figure 4.

The body thermophysiology was simulated with a metabolic rate of $70 \mathrm{~W} / \mathrm{m}^{2}$ (1.2 met) corresponding to an average office workload. The clothing has thermal resistance of $0.0945 \mathrm{~m}^{2} \mathrm{~K} / \mathrm{W}$ (0.61 clo) and vapor permeability of 0.41 , corresponding to average summer office dress code. The clothing area factor (ratio of clothed to naked surface) is 1.2.

\section{Numerical results}

The velocity and temperature field in the room are shown on Figure 5 and Figure 6 . The heat from the body can be seen to be advected towards the exhausts. Figure 7 shows the temperature distribution over the body surface, which is identical to the external clothing temperature. Not surprisingly, the front is cooler than the back, so the back has inhibited dry heat transfer and is expected to generate more latent heat loss (more sweating).

Figure 8 shows the dry heat flux density from the surface indicating that the arms and elbows, as well as the forehead, experience highest dry heat loss. The latent heat flux density is presented on Figure 9. The latent losses are generally low due to the relatively cool environment, yet they are considerable on the back due to the inhibited dry heat transfer from that body part.

Table 1 lists the integral dry and latent heat losses as well as heat flux densities from the body segments. The dry heat loss accounts for almost $80 \%$ of the losses. This ratio can change though with different activity and clothing.

Figure 10 shows the skin temperature distribution over

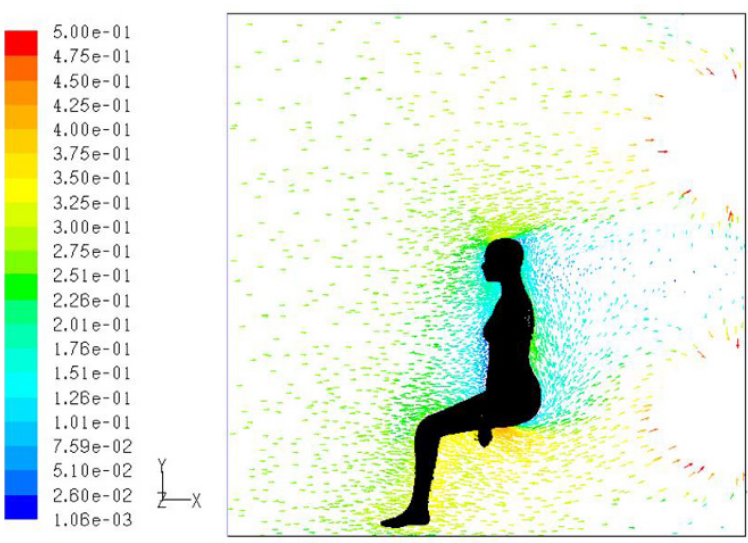

Figure 5. Velocity vectors in a midplane, coloured by magnitude $[\mathrm{m} / \mathrm{s}]$.
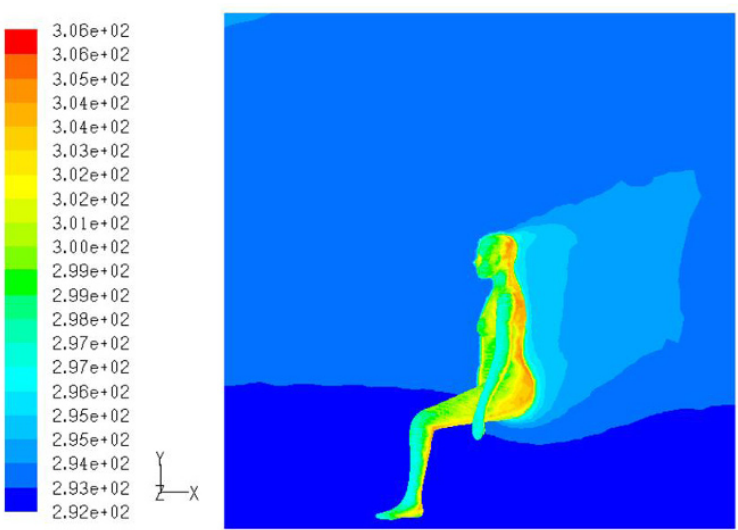

Figure 6. Temperature field in the midplane $[\mathrm{K}]$.

the body surface. It generally follows the trend of the clothing temperature (Figure 7), however it also depends on the dry heat flux according to equation (3). It can be noted that skin temperature exhibits smaller variations due to the protection of clothing. Body core temperature is determined by the iterative algorithm described before and is shown on Figure 11. Core temperature exhibits very small variations compared to skin due to the thermoregulatory control mechanisms of the body that try to maintain it within optimal range. Even a small increase of body core temperature triggers sweating, which is a powerful mechanism to increase heat loss, thus removing excess heat. Sweating is well related to a parameter called skin wettedness whose distribution 
Table 1. Predicted heat loss and heat flux density from the body segments.

\begin{tabular}{llllllll}
\hline $\begin{array}{l}\text { Body } \\
\text { segment }\end{array}$ & $\begin{array}{l}\text { Area } \\
\left(\mathbf{m}^{2}\right)\end{array}$ & $\begin{array}{l}\text { Dry heat } \\
\text { flux density } \\
{\left[\mathbf{W} / \mathbf{m}^{2}\right]}\end{array}$ & $\begin{array}{l}\text { Dry heat } \\
\text { Transfer } \\
{[\mathrm{W}]}\end{array}$ & $\mathbf{\%}$ & $\begin{array}{l}\text { Latent heat } \\
\text { flux density } \\
{\left[\mathbf{W} / \mathbf{m}^{2}\right]}\end{array}$ & $\begin{array}{l}\text { Latent heat } \\
\text { transfer } \\
{[\mathrm{W}]}\end{array}$ & \% \\
\hline back & 0.15 & 43.2 & 6.6 & 8.3 & 21.5 & 3.3 & 14.8 \\
chest & 0.15 & 54.5 & 8.4 & 10.6 & 12.9 & 2.0 & 9.0 \\
head & 0.09 & 52.1 & 4.7 & 5.9 & 14.3 & 1.3 & 5.8 \\
l_foot & 0.05 & 55.6 & 2.8 & 3.5 & 11.1 & 0.6 & 2.5 \\
I_forearm & 0.05 & 54.9 & 2.9 & 3.7 & 12.0 & 0.6 & 2.9 \\
I_hand & 0.03 & 55.9 & 1.4 & 1.8 & 11.1 & 0.3 & 1.3 \\
I_leg & 0.11 & 54.2 & 5.9 & 7.4 & 12.4 & 1.3 & 6.1 \\
I_thigh & 0.17 & 53.0 & 9.1 & 11.4 & 13.7 & 2.3 & 10.6 \\
I_upperarm & 0.07 & 52.5 & 3.7 & 4.6 & 13.6 & 1.0 & 4.3 \\
pelvis & 0.14 & 46.1 & 6.4 & 8.0 & 20.0 & 2.8 & 12.4 \\
r_foot & 0.05 & 55.7 & 2.9 & 3.6 & 11.0 & 0.6 & 2.5 \\
r_forearm & 0.05 & 55.1 & 3.0 & 3.8 & 12.0 & 0.7 & 3.0 \\
r_hand & 0.02 & 56.5 & 1.2 & 1.5 & 10.4 & 0.2 & 1.0 \\
r_leg & 0.11 & 54.3 & 6.0 & 7.6 & 12.4 & 1.4 & 6.2 \\
r_thigh & 0.17 & 52.9 & 9.2 & 11.5 & 13.7 & 2.4 & 10.7 \\
r_upperarm & 0.07 & 52.2 & 3.8 & 4.7 & 14.0 & 1.0 & 4.5 \\
tophead & 0.03 & 50.8 & 1.6 & 2.1 & 15.4 & 0.5 & 2.3 \\
\hline Net & 1.53 & 52.0 & $\mathbf{7 9 . 5}$ & $\mathbf{1 0 0 . 0}$ & $\mathbf{1 4 . 5}$ & $\mathbf{2 2 . 1}$ & $\mathbf{1 0 0 . 0}$ \\
\hline
\end{tabular}
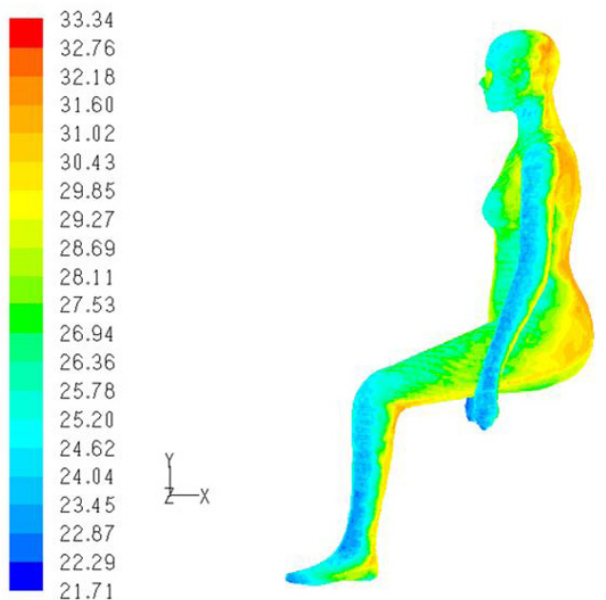

Figure 7. Temperature distribution over the clothed body $\left[{ }^{\circ} \mathrm{C}\right]$.

is shown on Figure 12. Low wettedness indicates regions with small latent heat loss and correlates well with the sensation of comfort. Skin wettedness cannot be less than 0.06 due to the natural uncontrollable diffusion of water through the skin dermal layer, and cannot exceed 1.0 which corresponds to completely wet body. Completely wet body does not ensure that all excess heat will be released, but that this heat will be the maximum available under the room conditions. This maximum depends on the

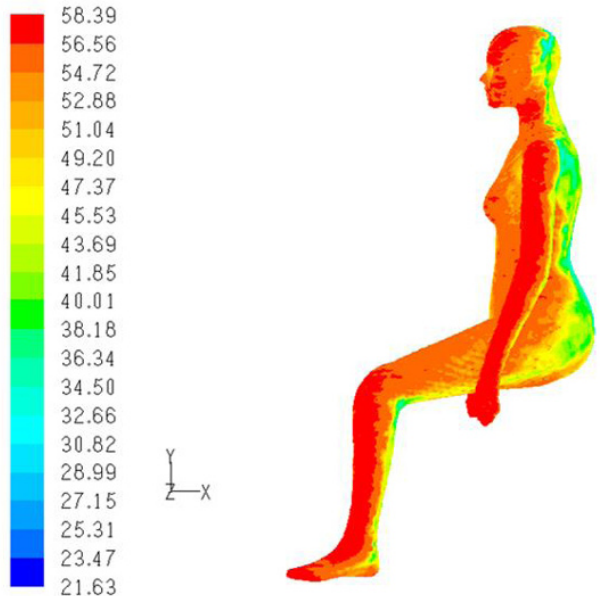

Figure 8. Dry heat flux density from the body surface $\left[\mathrm{W} / \mathrm{m}^{2}\right]$.

clothing characteristics and the water vapour potential between skin and room air. Highly humid air can drastically reduce the maximum available latent heat loss and the ability to cool the body via sweating, leading to increased skin weteddness and sensation of discomfort even at relatively low temperatures $\left(22-25^{\circ} \mathrm{C}\right)$. This may often be experienced at equatorial and tropical regions, or even after precipitations in spring or summer.

Finally, the distribution of humidity released from the skin 

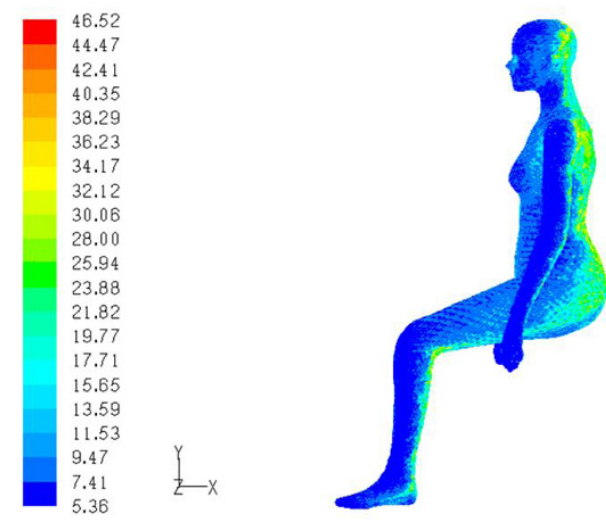

Figure 9. Latent heat flux density from the body surface $\left[\mathrm{W} / \mathrm{m}^{2}\right]$.
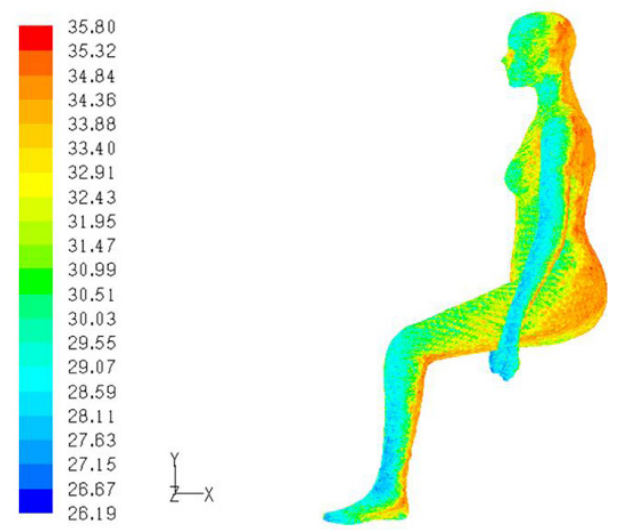

Figure 10. Skin temperature distribution over the body surface $\left[{ }^{\circ} \mathrm{C}\right]$.

was simulated and the results are shown on Figure 13. In order to obtain better results resolution for the distribution of humidity released from the body it was assumed that completely dry air enters the room. The back has highest percentage of latent heat loss (Table 1) and is therefore the biggest source of moisture. This moisture is advected by the flow towards the exhausts. Care was taken to specify a proper turbulent Schmidt number that determines the degree of moisture turbulent diffusion, which can be of the same order of magnitude as the convection. The origin of the moisture is mainly from sweat; therefore it can be viewed as an unpleasant pollutant or odour. Numerical simulation will reveal whether it is removed efficiently from the room without much interaction with other occupants. The moisture

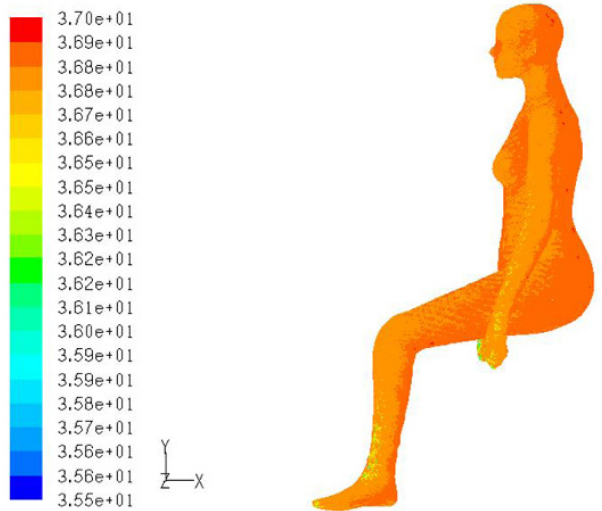

Figure 11. Body core temperature $\left[{ }^{\circ} \mathrm{C}\right]$.

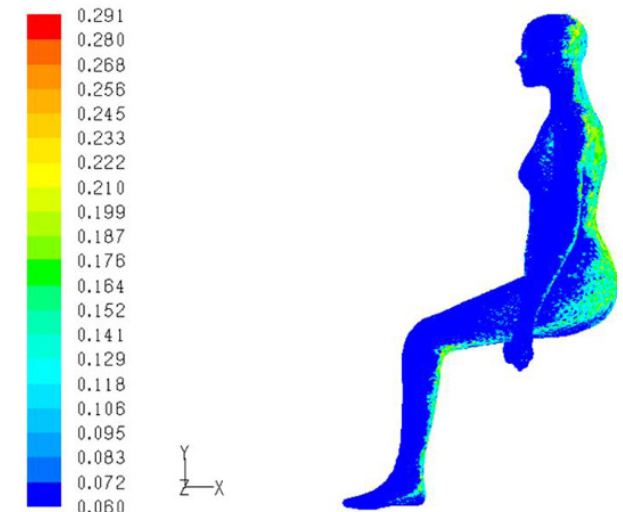

Figure 12. Skin wettedness [-].

field can also be used to evaluate some of the scales for ventilation efficiency (SVEs) developed by [9].

\section{Physical experiment}

To validate the numerical results, data from a laboratory experiment conducted by [10] are used. A naked thermal mannequin with a controlled surface temperature of $34^{\circ} \mathrm{C}$ is positioned in the middle of the room. The experiment measures the velocity and temperature profiles at chosen locations along with the heat loss from each individual segment of the mannequin. The location of the mannequin and measurement positions are shown on Figure 14. The air velocity is measured at 5 vertical points behind the 


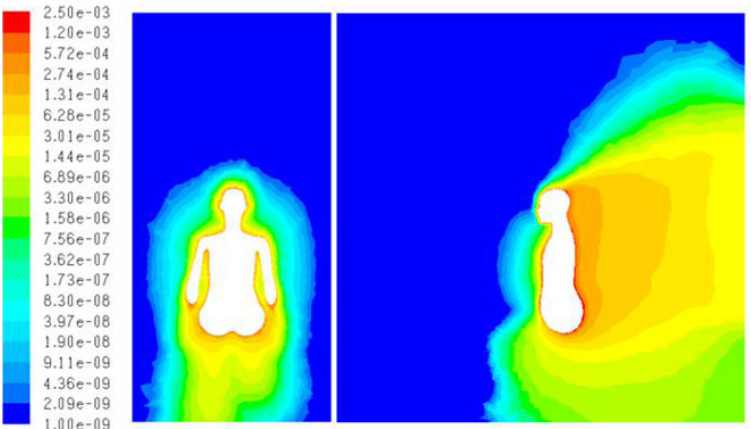

Figure 13. Absolute humidity $[\mathrm{kg} / \mathrm{kg}]$

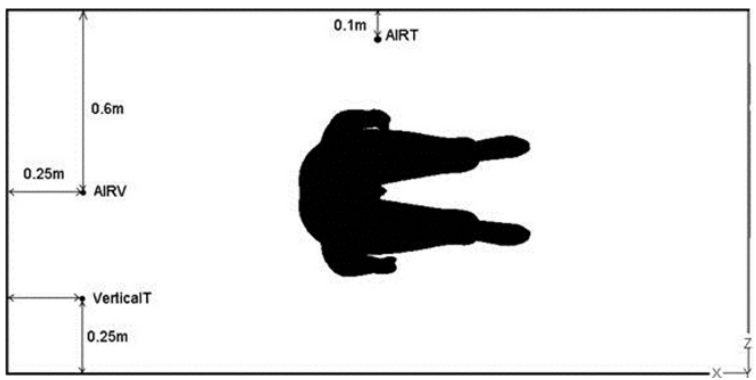

Figure 14. Location of mannequin and measurement positions.

mannequin, (position AIRV). Temperatures are measured in 4 points to the left of the mannequin $0.1 \mathrm{~m}$ from the wall (position AIRT) and behind the mannequin (position VerticalT).

Figure 15 shows the temperature profile in position AIRT (for measurement locations refer to Figure 14). The simulated temperature profile agrees well with the measurements, with mean absolute error of $0.16^{\circ} \mathrm{C}$. The lowest measurement point has a measurement value lower than the supply air temperature range $\left(19.6^{\circ} \mathrm{C}\right.$ to $\left.20.8^{\circ} \mathrm{C}\right)$ and is very unlikely to occur; especially in the presence of a heat source like the thermal mannequin. It is possible that this value lies outside the feasible temperature range of the experiment.

Figure 16 presents the temperature profile measured behind the mannequin back (position VerticalT). The agreement between measurements and simulations is excellent again, with mean absolute error of only $0.1^{\circ} \mathrm{C}$.

Figure 17 shows comparison of measured and simulated velocity magnitude in position AIRV. The agreement is excellent, and discrepancy is mostly evident for higher

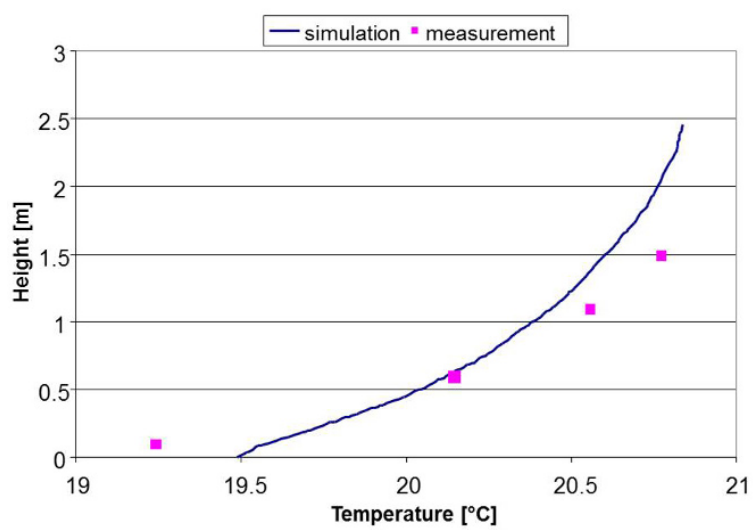

Figure 15. Temperature profile in position AIRT $(X=1.22 \mathrm{~m}, \mathrm{Z}=$ $1.1 \mathrm{~m}$ ). Measurement data taken at heights $Y=0.1,0.6$, $1.1,1.5 \mathrm{~m}$.

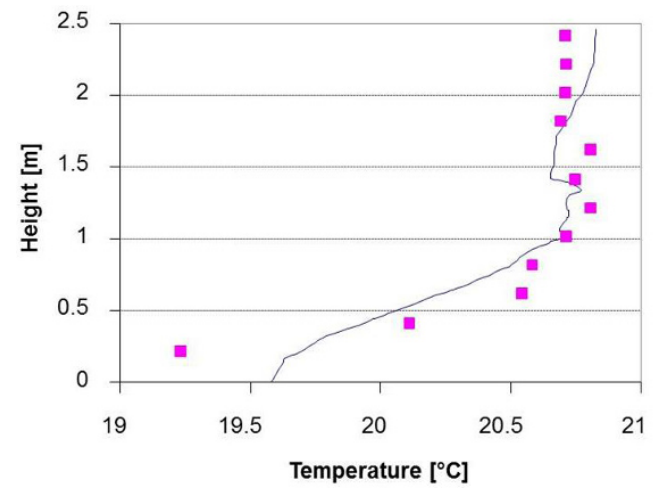

Figure 16. Temperature profile in position VerticalT $(X=2.19 \mathrm{~m}, \mathrm{Z}$ $=0.25 \mathrm{~m}$ ). Measurement data taken at heights $Y=0.2$, $0.4,0.6,0.8,1,1.2,1.4,1.6,1.8,2,2.2,2.4 \mathrm{~m}$.

heights, where the measured values exceed the predicted. A similar result is reported in [11], the author of which compared his numerical predictions with these measurements. It is a general observation that the measured velocity exceeds the predicted velocity due to the way velocity magnitude is calculated in CFD, and the discrepancy is larger with larger turbulence intensity [12]. This correlates very well with the case here since, according to measurement data, the higher points do have higher turbulence intensity. This explains the relatively large mean absolute error of $0.15 \mathrm{~m} / \mathrm{s}$ and a normalized mean bias error [13] of $27 \%$ for this variable.

Figure 18 presents the heat flux density from the 16 mannequin segments, both measured and simulated. The 


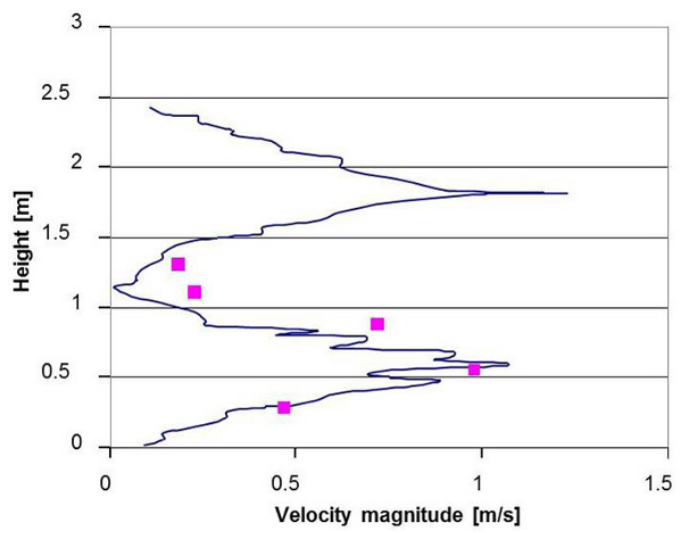

Figure 17. Velocity profile in position AIRV. Measurement data taken at heights $Y=0.275 \mathrm{~m}, 0.550 \mathrm{~m}, 0.875 \mathrm{~m}, 1.100 \mathrm{~m}$ $1.300 \mathrm{~m}$.

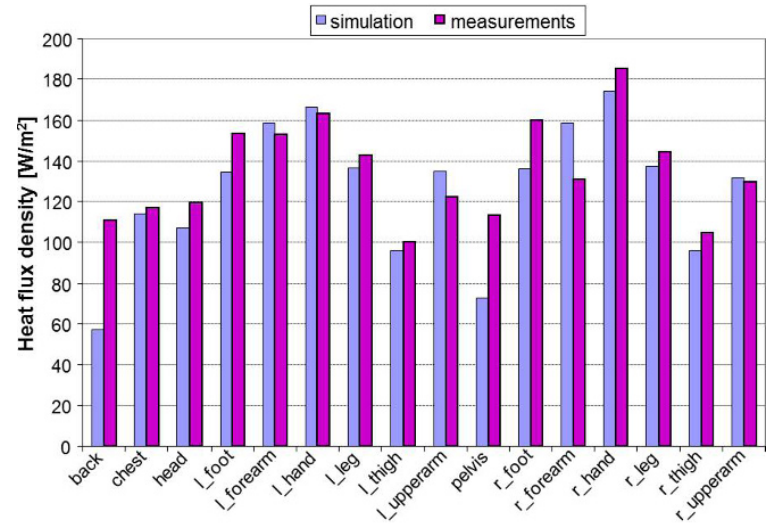

Figure 18. Simulated and measured heat flux density from the segments of the mannequin.

mean absolute error was $16 \mathrm{~W} / \mathrm{m}^{2}$, while the normalized mean bias error was only $5 \%$. Figure 19 shows the relative error between the heat flux densities over all mannequin segments. This error is completely acceptable with the exception of the back and pelvis, which are the segments with violated $y^{+}$condition. This condition requires too fine a computational mesh to satisfy and would lead to prohibitively long computational time on the available computer hardware. Nevertheless, the numerical results positively illustrate the need to comply with the turbulence model's requirements as they closely correlate to the measurements when fulfilled.

In summary, the comparison between measurements and

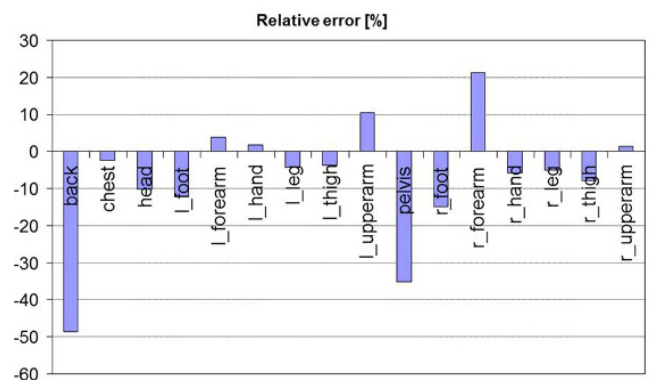

Figure 19. Relative error [\%] between simulated and measured heat flux over the mannequin segments.

simulations has demonstrated good performance of the numerical predictions with few exceptions. The first discrepancy is where dimensionless wall distance was not maintained, and the second discrepancy is with a measurement value possibly lying outside the feasible range of the physical experiment.

\section{Conclusions}

The inclusion of a two-node thermo-physiological model to represent the human body in a CFD simulation provides more realistic boundary conditions for the numerical simulation of the room airflow. The dry and latent heat resistance of the clothing was also taken into consideration. The model can predict the local temperature distribution over the skin, the dry and latent surface heat loss, skin wettedness, body core temperature, and other relevant thermophysiological parameters. The stability of the computational procedure is ensured by employing under-relaxation for the latent heat flux as well as indirectly to the dry heat flux. Increased latent heat flux was correctly predicted in areas with inhibited heat transfer due to poor convection. The two-node model is robust and can be recommended for use in simple applications where high accuracy of thermal body stress prediction is not pursued.

\section{References}

[1] Stolwijk J. A .J., Hardy J .D., Temperature regulation in man-A theoretical study, Pflugers Archiv, 1966, 291

[2] Gagge A. P., Stolwijk J. A, Nishi Y., An effective temperature scale based on a simple model of human physiological regulatory response, ASHRAE Trans., 
1970, 77(1), 247-262

[3] ASHRAE Fundamentals Handbook, Chapter 8, 1993

[4] Murakami S., Kato S., Zeng J., Combined simulation of Airflow, Radiation and Moisture transport for Heat Release from Human Body, Building and environment, 2000, 35(6), 489-500

[5] Cheng Y., Niu J., Gao N., Thermal comfort models: A review and numerical investigation, Building and Environment, 2012, 47, 13-22

[6] Fiala D., Dynamic Simulation of Human Heat Transfer and Thermal Comfort, PhD thesis Institute of Energy and Sustainable Development, De Montfort University Leicester, 1998

[7] van Treeck C., Frisch J., Pfaffinger M., Rank E., et. al. 2009. Int. thermal comfort analysis using a parametric manikin model for interactive simulation. J Building Performance Simulation, 2009, 2(4):233-250

[8] Pichurov G., Gagge model, Tanabe model - Integration of CFD, Proc. $5^{\text {th }}$ Int. Course "Climacademy", 16-23 October, 2008, Pamporovo Bulgaria, 2008, 47-51, (http://www.climacademy. tu-sofia.bg/course5.php)

[9] Kato S., Murakami S., Kobayashi H., New scales for evaluating ventilation efficiency as affected by supply and exhaust openings based on spatial distribution of contaminant. In: Murakami S., et al. (Eds), Room Air Convection and Ventilation Effectiveness (ISRACVE Proceedings), 1993

[10] Nielsen P. V., Murakami S., Kato S., Topp C., et al., Benchmark Tests for a Computer Simulated Person, ISSN 1395-7953 R0307, Department of Building Technology and Structural Engineering, Aalborg University, 2003, http://cfd-benchmarks.com/

[11] Yang T., Cropper P. C., Cook M. J., Yousaf R., et al., A new simulation system to predict human-environment thermal interactions in naturally ventilated buildings, Proc $10^{\text {th }}$ Int Building Performance Simulation Association (IBPSA) Conf 2007, 03-06 Sept, Tsinghua Univ, Beijing, China, 2007, pp 751-756, ISBN: 0-9771706-2-4 THERMAL INTERACTIONS IN NATURALLY VENTILATED BUILDINGS

[12] Koskela H. Modelling thermal comfort in office environment, Proc. $5^{\text {th }}$ Int. Course "Climacademy", 16-23 October, 2008, Pamporovo Bulgaria, ISBN 978-954-92261-3-3, 2008, 41-46, (http://www. climacademy.tu-sofia.bg/course5.php)

[13] ASHRAE guideline 14-2002, Measurement of energy and demand savings, 2002 